FACULDADE DE CIÊ NCIAS ECONÔ MICAS DA UFRGS

REESTRUTURAÇÁ O DA PRODUÇÁ O AGRICOLA NO RIO GRANDE DO SUL, DE 1970 A 1996 E UMA ESTIMAÇȦ O PARA 2012 TIAGO WICKSTROM ALVES

A CRIMINALIDADE NA RECIAO METROPOLTANA DE SALVADOR JOSÉ CARRERA FERNANDEZ, LUIZ FERNANDO LOBO

COMPEIITIVIDADE INTERNACIONAL EM SOFTWARE: UM ESTUDO SOBRE A EXPERIÊNCIA DE FLORIANÓ POLIS HOVÉDO NUNES LINS

MODELOS DE ORGANIZAÇẢ O E REPARTIÇẢ O DE RENDAS NA CADEIA PRODUTIVA DO GÁS NATURAL.

HELder QUEIROZ PINTO Jú NIOR, RODOLFO TORRES dOS SANTOS

REGIMES MONETÁ RIOS E DIVIDA PÚ BLICA: UMA ANÁ LISE DE MECANISMOS ALTERNATIVOS DE COORDENAÇÃO MACROECONÓ MICA

manoel Carlos de Castro Pires

ECONOMIA DOS RECURSOS NATURAIS E SEUS INDICADORES DE ESCASSEZ: UMA QUESTÃ O DE SUSTENTABILIDADE ANDREA SALES SOARES DE AZEVEDO MELO

O ECOPROTECIONISMO AGRICOLA NA UNIĀ O EUROPEIA E SEUS POSSIVEIS IMPACTOS SOBRE A ECONOMIA BRASILEIRA Rodrigo daniel Feix, Clá udio R. Fóffano Vasconcelos

PADRÓ ES DE DESENVOLVIMENTO, FUNÇ Ó ES ESTATAIS E ENDIVIDAMENTO NO CAPITALISMO CONTEMPORÁNEO paulo Balanco, Eduardo Costa Pinto

A INTERNACIONALIZAÇÃO DO VAREJO A PARTIR DOS CASOS WAL-MART E CARREFOUR

Armando joảo dalla Costa

UMA ANÁ LISE ECONOMÉTRICA DO FUTEBOL BRASILEIRO ARI Francisco de Araujo JR, Clá udio D. SHIKIDA, LEONARDO M. MONASTERIO

O BRASIL E A ECONOMIA INTERNACIONAL: RECUPERAÇÃ O E DEFESA DA AUTONOMIA NACIONAL

RESENHA DE ALEXANDRE CÉ SAR CUNHA LEITE

REGIMES MONETÁ RIOS: TEORIA E A EXPERIÊNCIA DO REAL ResenHa de Fernando Ferrari Filho

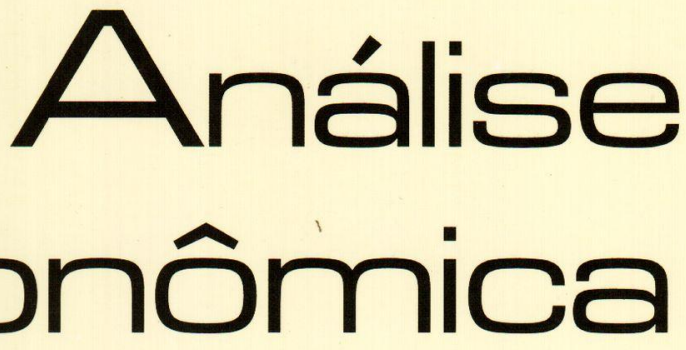


Universidade Federai. do Rio Grande do Sul Reitor: Prof José Carlos Ferraz Hemnemann

Facul dade de Cuéncias Economicas

Diretor: Prof. Gentil Corazza

Ceniro de Estudos f Prequisas Economicas

Diretor: Prof. Lovois de Andrade Miguel

Depariamenio de Crências Economicas

Chefe: Prof Ricardo Dathein

Departamento de Cîncias Coniábeis f A tuaria Chefe: Prof. Ceno Odilo Kops

Curso de Pós-Graduaçó em Economia

Coordenador: Prof. Fernando Ferrari Filho

Curso de Pos-Grainaça em Desenvolvimento RuRAI

Coordenador: Prof. Paulo Dabdab Waquil

Conseluo Eni tokial: André Moreira Cunha (UFRGS) Carlos G. A. Mielitz Netto (UFRGS), Carlos Henrique Hom (UFRGS). Eduardo A. Maldonado Filho (UFRGS). Eleutério F S. Prado (USP), Eugênio Lagemann (UFRGS), Fernando Cardim de Carvalho (UFRJ). Femando Ferrari Filho (UFRGS), Fernando de Holanda Barbosa (FGV/RJ), Flávio Augusto Ziegelmann (UFRGS), Flávio Vasconcellos Comin (UFRGS), Gentil Corazza (UFRGS), Giácomo Balbinot to Neto (UFRGS), Gustavo Franco (PUC/RJ) Hélio Henkin (UFRGS), Jan A. Kregel (UNCTAD), João Rogério Sanson (UFSC), Joaquim Pinto de Andrade (UnB), Júlio César Oliveira (UFRGS), Luiz Estrella Faria (UFRGS), Luiz Paulo Ferreira Nogueról (UFRGS), Marcelo S Portugal (UFRGS), Maria Alice Lahorgue (UFRGS), Octávio Augusto Camargo Conceicão (UFRGS), Paul Davidson (University of Tennessee), Paulo D. Waquil (UFRGS), Pedro C. D. Fonseca (UFRGS), Philip Arestis (University of Cambridge), Ricardo Dathein (UFRGS) Ronald Otto Hillbrecht (UFRGS), Sabino da Silva Porto Jr (UFRGS), Sérgio M. M. Monteiro (UFRGS), Stefano Florissi (UFRGS) e Werner Baer (University of IIIinois at Urbana - Champaign)

Comissão Emroniai: Eduardo Augusto Maldonado Filho, Fernando Ferrari Filho, Hélio Henkin. Marcelo Savino Portugal, Paulo Dabdab Waquil e Sérgio Marley Modesto Monteiro.

EnIrok: Sérgio Marley Modesto Monteiro

Ediror Adjun ro: Hélio Henkin

Secretário: Emerson Douglas Neves

REVISÃo DE IEX ros: Vanete Ricacheski

Emioração: Núcleo de Editoração e Criação da

Gráfica da UFRGS - Janaína Horn e Junia Saedt

Fundador: Prof Antonio Carlos Santos Rosa
Os materiais publicados na revista Análise Econômica são da exclusiva responsabilidade dos autores. É permitida a reprodução total ou parcial dos trabalhos, desde que seja citada a fonte. Aceita-se permuta com revistas congêneres. Aceitam-se, também, livros para divulgacão, elaboraça de resenhas e recensões Toda correspondência: material para publicação (vide normas na terceira capa), assinaturas e permutas devem ser dirigidos ao seguinte destinatário:

Prof. Sérgio Marley Modesto Monteiro Revista Andilse Económica - Av. João Pessoa, 52 CEP 90040-000 PORTO ALEGRE - RS, BRASIL Telefones: (051) $33163513 / 33164164$

Fax: (051) 33163990 - E-mail: rae@vortex ufrgs.br

A Revista Análise Econômica agradece a colaboração dos pareceristas do número 43, abaixo relacionados:

Adalmir Antonio Marquetti

André Moreira Cunha

Carlos José Caetano Bacha

Carlos Mielitz. Netto

Clailton Ataídes de Freitas

Claudio Roberto Fóffano Vasconcelos

Eduardo Ernesto Filippi

Eduardo Pontual Ribeiro

Eleuterio Fernando da Silva Prado

Fernando J Cardim de Carvalho

Fernando Augusto Mansor de Mattos

Fernando Ferrari Filho

Flávio Tosi Feijó

Flavio Vilela Vieira

Francisco Paulo Cipolla

Gentil Corazza

Gíacomo Balbinotto Neto

Helio Henkin

Jefferson Andronio Ramundo Staduto

João de Deus Sicsú Siqueira

José Carrera Fernandes

Leonardo Monteiro Monasterio

Luís Fernando de Paula

Luis Roberto Nascimento

Manoel Carlos de Castro Pires

Octavio Augusto Camargo Conceição

Patrízia Raggi Abdallah

Robson Antonio Grassi

Ronald Otto Hilbrech

Tiago Wickstrom Alves

Tito Belchior Silva Moreira

Análise Econônica

Ano 23, n ${ }^{\circ} 44$, setembro, 2005 - Porto Alegre

Faculdade de Ciências Economicas, UFRGS, 2000

Periodicidade semestral, março e setembro

1. Teoria Econômica - Desenvolvimento Regional -

Economia Agrícola - Pesquisa Teórica e Aplicada -

Periódicos. I.. Brasil

Faculdade de Ciências Econômicas,

Universidade Federal do Rio Grande do SuI

CDD 330.05

CDU $33(81)(05)$ 


\section{Economia dos recursos naturais e seus indicadores de escassez: uma questão de sustentabilidade}

Andrea Sales Soares de Azevedo Melo*

Resumo: A ciência econômica divide seu campo de estudo para as questões do meio ambiente a partir das funções que este exerce na vida do homem. Neste contexto, a Economia dos Recursos Naturais trata o mesmo como ofertante de recursos, o que, tradicionalmente, foi sempre trabalhado em termos de eficiência intergeracional. O desenvolvimento sustentável, entretanto, provocou que a disponibilidade de recursos passasse a ser vista como uma questão de eqüidade, e não mais de eficiência, fato que representa uma mudança de paradigma para a Economia dos Recursos Naturais. Este artigo faz uma resenha do estado da arte neste campo da economia, ressaltando os principais pontos que se encontram atualmente em discussão, em especial no que se refere ao desenvolvimento de indicadores da escassez da disponibilidade dos recursos.

Palavras-chave: sustentabilidade, economia dos recursos naturais, indicadores de escassez.

Abstract: Economic science has, at least, three ways of leading with environmental questions, depending on the function the environment plays at man's life. Natural resource economics treats the environments as a resource supplier and has traditionaly worked it in terms of inter-generational efficiency. However, sustainable development has provoked change in paradigms: the availability of the resources became an equity subject, instead of only a question of efficiency. This article makes a review of the state of the art in this field of the Economy, hilighting the mais points that are currently in discussion, especially referring to the development of indicators of the resources scarcity.

Keywords: sustainability, natural resource economics, scarcity indicators.

JEL: Q56.

1 Introdução

A ciência econômica enxerga o meio ambiente através das funções que este ambiente desempenha na vida do homem, ou seja, o entende como um meio para a satisfação das necessidades humanas. Segundo Naess (1990), esta é a principal característica do movi-

* Professora do Departamento de Economia da UFPE. 
mento ambientalista surgido na década de 1980; os ambientalistas mais antigos, da deep ecology, não atribuíam superioridade do homem sobre as outras espécies.

Sob este prisma, pode-se destacar que a economia reconhece três funçōes básicas do meio ambiente. A primeira dessas funções é a de fonte de amenidades, ou de lazer. Segundo Fischer e Peterson (1976), foi John Stuart Mill, em 1865, que, ao ressaltar a importância do ambiente para a qualidade de vida (como depositário de solitude e beleza natural), deu origem ao estudo do ambiente como fonte de amenidades na economia.

A segunda função reconhecida é a de receptor dos dejetos das atividades de produção e consumo. A sua capacidade de degeneração dos dejetos e de sua própria regeneração é extremamente importante e garante a sobrevivência de muitas espécies, além da própria qualidade do ar, água, solo, entre outros recursos.

A terceira e última função do meio ambiente é a de ofertante de recurso natural, o principal insumo da atividade produtiva. Em se tratando da escassez desses recursos, Smith e Ricardo são importantes nomes a se lembrar, por terem sido os primeiros a chamar a atenção para a escassez absoluta e relativa, respectivamente, do recurso terra.

Todas estas três funções são igualmente importantes e altamente correlacionadas entre si; daí a natureza holística da questão ambiental. A intervenção em qualquer uma delas pode provocar o desequilíbrio em outra, ou mesmo no ambiente como um todo.

Tem-se tornado senso comum considerar a Economia do Meio Ambiente como a parte que estuda as duas primeiras funçōes; enquanto que a Economia dos Recursos Naturais é a que trata das nuanças relativas à ultima função. Existe, no entanto, alguma discordância no que se refere a esta divisão, uma vez que se entende que a Economia do Meio Ambiente deveria abarcar todas as funções (SOUZA, 1997); mas este tem sido o trabalho da Economia Ecológica.

Perceba-se, todavia, que tratar o ambiente por inteiro não significa simplesmente a soma de suas partes, ou funções, dado que estas têm entre si uma relação desconhecida e provavelmente não-linear. ${ }^{1} \mathrm{E}$, ainda, que o fato de se dividir as suas funções para estudo não significa que na Economia dos Recursos Naturais, por exemplo, não se considerem os efeitos poluentes de uma atividade extrativa qualquer.

O desenvolvimento das duas primeiras economias tem-se dado de forma sistemática desde 1967, instigado por John V. Krutilla. Naquele momento, Krutilla já encontrava razōes para que os retornos

1 Para saber o quão complexos podem ser os modelos que tratam dessa inter-relação ver, por exemplo, Batabyal, Kahn e O'neill (2003). 
privados e sociais do uso do ambiente divergissem significativamente, sugerindo que a análise econômica tradicional não se adaptava completamente ao caso. Este reconhecimento é identificado como determinante e propulsor do desenvolvimento dos estudos referidos.

Entretanto, apenas a partir do princípio dos anos 1980, com o advento da World Cconservation Strategy (WCS), e da World Comission on Environment and Development (WCED), já em 1987, devido aos esforços mais contundentes no sentido de "desmistificar" a questão ambiental dentro do processo de desenvolvimento econômico, que o meio ambiente em economia tem tido uma grande evolução, com o surgimento e difusão da Economia Ecológica, e com a evolução da Economia do Meio Ambiente e dos Recursos Naturais. Ou seja, é a divulgação e evolução do conceito de desenvolvimento sustentável que tem trazido evolução e divulgação para o meio ambiente na economia.

Para a Economia dos Recursos Naturais deve-se destacar ainda outro marco, que é o do documento do Clube de Roma Limits to Growth, de 1972. O principal ponto ressaltado por este documento àquela época foi o da finitude dos recursos naturais e, por isso, a existência de limites naturais para o crescimento econômico; principalmente quando se considera a expansão dos países mais pobres. Mas é possível que se diga que os próprios movimentos de desenvolvimento sustentável foram instigados pelo Clube de Roma, muito embora tenham mudado um pouco a visão. Segundo Shi (2004), o recurso natural deixa de ser visto como um limite para o crescimento econômico, para ser visto como elemento de eqüidade entre as gerações.

Enfim, foi desse movimento de desenvolvimento sustentável que surgiu a idéia de conciliação (pelo menos teoricamente) entre ambientalistas (ecologistas) e desenvolvimentistas (economistas) (LÉLÉ, 1991; GOMES, 1995 133CARRERA-FERNANDEZ e PEREIRACARRERA-FERNANDEZ e PEREIRAA MARSDEN, 1994). Sustentabilidade, como vista pela "Comissão Mundial", ${ }^{2}$ adiciona considerações ambientais ao desenvolvimento econômico, buscando fazer convergir esses interesses supostamente díspares. $\mathrm{E}$, ao fazer isso, modifica o próprio entendimento padrão de desenvolvimento, incorporando a este entendimento considerações de eqüidade intergeracional. ${ }^{3}$

A proteção ambiental está colocada no Relatório Brundtland ${ }^{4}$

2 Comissão da ONU para discussão de assuntos de sustentabilidade: WCED.

3 Muito embora já se possa perceber desde 1977, com os trabalhos de Hartwick (Hartwick, 1977), a preocupação com a eqüidade intergeracional.

${ }^{4}$ Relatório Brundtland foi o documento produzido no âmbito da WCED (WCED, 1987). 
como uma medida necessária para garantir os interesses das gerações futuras, as quais herdarão o mundo natural como seu legado. Isto é, a proteção ambiental é importante para construir uma nova categoria de eqüidade, a qual é conhecida como eqüidade intergeracional: as necessidades das gerações atuais têm de ser satisfeitas sem comprometer a habilidade das futuras gerações de satisfazerem suas próprias necessidades.

Desenvolvimento sustentável traz, então, um novo problema para a teoria econômica, a qual vinha discutindo a questão intergeracional apenas em termos de eficiência. ${ }^{5}$ Segundo Howarth e Norgaard (1994), eqüidade e eficiência são duas coisas bastante diferentes, sendo que a primeira implica um conjunto muito mais restrito do que segunda, no sentido de que a conservação ambiental deve se dar de forma muito mais conservadora quando se leva em consideração não só a eficiência, mas também a eqüidade. Sendo assim, concluem os autores, limites de fora do mercado devem ser impostos para que a sustentabilidade possa se realizar, ou seja, através da mão do Estado. O âmbito da atuação do Estado neste caso deve se dar sobre todas as funções do meio ambiente na vida do homem, garantindo que ocorra o crescimento econômico, mas sob os limites da conservação ambiental.

Em resumo, o desenvolvimento sustentável veio impor um padrão de uso do ambiente diferente daquele imposto pelo padrão de desenvolvimento econômico e, por isso, a teoria econômica precisaria evoluir para conter esta questão. Outrossim, Rammel e van den Berg (2003) argumentam que as políticas sugeridas pela teoria econômica padrão não estimulam o desenvolvimento sustentável. Para isto, segundo os autores, é preciso que se use uma perspectiva evolucionária, "caracterizada pela diversidade, seleção, inovação, caminhos dependentes e racionalidade restringida".

Neste contexto, este artigo tem como objetivo levantar uma das principais questões surgidas no âmbito da Economia dos Recursos Naturais, que é a da medição da eqüidade em termos da disponibilidade dos recursos, ressaltando conceitos e medidas de escassez. Não pretende exaurir a questão ambiental dentro da economia, posto que esta é muito ampla e inexaurível, mas apenas apresentar alguns problemas sobre os quais os pensadores se debruçam na atualidade.

As seções seguintes foram pensadas buscando uma orientação didática para este estudo, divididas da seguinte forma. A seção 2esclarece conceitos e terminologias, trazendo a identificação de tipos e classes de recursos naturais. A seção 3 traz à discussão a teoria, os

5 Que significa uma distribuição eficiente de recurso entre as gerações. 
elementos e os instrumentos atualmente utilizados na economia, para tratar a escassez do recurso, os quais são importantes em ambiente de desenvolvimento sustentável e equidade intergeracional. E, por fim, a seção 4 traz as considerações finais.

\section{A taxonomia dos recursos}

Tietenberg (1994) sugere a classificação dos recursos naturais de três formas diferentes. A primeira, que discrimina a disposição dos recursos em sua forma natural e acessibilidade; a segunda, que discrimina o fluxo de renovação deste recurso na natureza; e a terceira, que divide os recursos entre recursos recicláveis e não-recicláveis.

Quanto à acessibilidade

São usados três conceitos para classificar os recursos quanto à sua acessibilidade: dotação de recursos, reservas potenciais e reservas correntes.

A Dotação de Recurso é a ocorrência natural do recurso na terra. ${ }^{6}$ É um conceito geológico, já que nem toda dotação de recurso existente na crosta terrestre pode ser considerada como disponível para a atividade econômica, dada a sua inacessibilidade em alguns casos. Sendo assim, é o limite superior da disponibilidade do recurso.

As Reservas Potenciais, por sua vez, são melhor expressas por uma função, que tem como variável independente o preço: $R_{\text {pot }}=$ $\mathrm{f}(\mathrm{p})$; onde $\mathrm{R}_{\mathrm{pot}}$ é a reserva potencial e $\mathrm{f}(\mathrm{p})$ uma função do preço do recurso.

Refere-se esta reserva àquela parte da dotação de recursos que, para sua exploração, depende do preço que o mercado esteja disposto a pagar. É uma relação positiva, significando que, quanto maior o preço, maior a reserva potencial. Preços maiores justificam os maiores gastos necessários para a exploração de determinadas reservas, de dificil acesso e, portanto, demandantes de uma tecnologia mais sofisticada. Imagine-se, neste caso, um poço de petróleo tão profundo que os gastos necessários para alcançá-lo não valem a pena, dado que os preços de mercado pagos por este não chegarão a cobrir os custos incorridos; isto é, pelo menos até que a necessidade leve à utilização deste poço.

Já as Reservas Correntes, por sua vez, têm seu tamanho definido como um número; é uma quantidade que existe, independentemente

\footnotetext{
- Isto enquanto a dotação do recurso em outros planetas ou sistemas não estiverem fazendo parte da definição.
} 
de qualquer outra variável. As reservás correntes se referem aos recursos que já são conhecidos e que podem e estão sendo extraídos de forma lucrativa, através das ações do mercado. A Figura 1 a seguir retrata um pouco a diferença destas três classes.

$O$ correto entendimento e diferenciação destas três classes de reservas é extremamente importante quando se fala de exaustão de um recurso, posto que exaurir a reserva potencial deste é muito diferente de exaurir a sua reserva corrente. A exaustão referida no primeiro caso é um resultado definitivo, sem volta, irreversível, já que se considera que a diferença entre esta reserva e a dotação do recurso é completamente inacessível. No segundo caso é apenas uma questão de tempo e de inovação tecnológica, dado que a diferença entre as duas reservas é preço, que, ao se elevar, pode tornar mais uma parte da reserva potencial como reserva corrente.
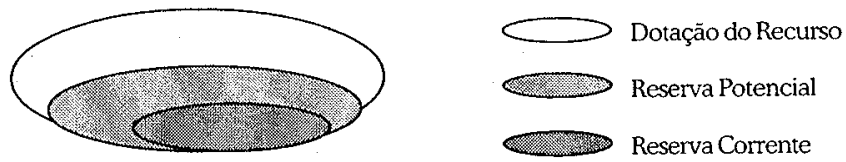

Figura 1: Representação das três classes de reservas de recurso natural. Fonte: Elaboração própria

Outra questão importante, pelo que acabou de ser exposto, é que consiste de um erro grave considerar a dotação de recurso como uma reserva potencial. Neste caso, destacam-se as visōes muito otimistas de perspectivas de inovação tecnológica, geralmente ligadas aos economistas.

\section{Quanto à taxa de renovação}

Quanto à taxa de renovação, os recursos podem ser esgotáveis, ou não-renováveis; e não esgotáveis, ou renováveis. Um recurso esgotável é um recurso cuja reposição natural pode ser ignorada; a taxa de reposição deste recurso é tão baixa que ela não oferece um aumento de "reserva potencial", num horizonte de tempo significativo para a vida humana.

A dotação de recursos esgotáveis é de tamanho finito e o seu uso necessariamente leva à diminuição da reserva. Os minérios de uma forma geral são recursos esgotáveis. 
Os recursos renováveis, por sua vez, têm uma taxa de reposição natural que é significativa, de forma que sua dotação de recursos pode ser considerada infinita, desde que respeitada esta taxa de renovação. Floresta, água, energia solar, a vida animal, entre outros, são considerados recursos renováveis.

\section{Quanto à reciclagem}

Os recursos naturais podem ser recicláveis ou não. Um recurso reciclável é aquele cuja massa pode ser recuperada, quando, na sua forma original, não despertar mais interesse em seu uso. Um recurso não-reciclável é aquele cuja massa não pode ser recuperada.

Existem certamente recursos que jamais poderão ser reciclados, como a energia. Todavia, a variável preço é determinante neste processo, significando que, em sendo muitos recursos potencialmente recicláveis, estes só o serão se o custo de reciclagem for compensatório, ou seja, se existir mercado para ele. É tudo uma questão de tempo até que se justifique a reciclagem: ou tempo do preço do produto se elevar, devido à sua exaustão iminente; ou de haver um avanço tecnológico que diminua o custo referido.

Perceba-se que pode haver um intenso jogo entre reciclagem e recursos esgotáveis, de forma que a reserva potencial do recurso diminua numa velocidade cada vez menor no tempo. Entretanto, necessariamente haverá uma diminuição desta reserva no tempo, já que a lei da entropia, física, garante que um recurso não possa ser $100 \%$ reciclado.

O papel que a reciclagem desempenha na economia e na preservação do recurso natural é importantíssimo, e depende se este recurso é renovável ou não. Em princípio, se um recurso for renovável e se sua utilização se der em respeito à sua taxa de renovação, a reciclagem não será necessária. A taxa de utilização do recurso renovável, no entanto, também depende do avanço tecnológico, que pode se dar de forma mais lenta do que o avanço tecnológico na área da reciclagem do recurso. Existe entre estes dois pontos, certamente, uma situação de equilíbrio dinâmico, o qual se altera com o tempo, a escassez, os preços dos recursos e do recurso reciclado e com o avanço tecnológico nas duas áreas.

\section{Conceitos e indicadores de escassez}

O conceito de sustentabilidade é hoje dividido em duas classes: sustentabilidade forte e sustentabilidade fraca. ${ }^{7}$ No primeiro caso tem-

\footnotetext{
7 Veja para maiores detalhes HOWARTH (1996).
} 
se a visão mais conservadora no que se refere à conservação do meio ambiente, sendo que cada recurso é único e deve ser visto como insubstituível; no segundo caso, a sustentabilidade é vista de forma mais ampla, no agregado ambiental, em que se considera importante o valor total do ambiente como um todo, e não cada recurso individualmente. Ou seja, se um recurso tiver chegado à exaustão, mas tiver sido substituído por outro em suas funçōes econômicas, então, ainda assim, existe sustentabilidade. ${ }^{8}$

De qualquer forma, seja qual for o conceito de sustentabilidade utilizado como padrão ideal de comportamento, forte ou fraco, a disponibilidade dos recursos precisa ser monitorada, para medir a sua tendência no tempo. Afinal, na vida real, existem evidências de que o desenvolvimento sustentável não está sendo alcançado9 (ANDRIANTIATSAHOLINIAINA, KOUIKOGLOW e PHILLIS, 2004). Outrossim, Fisher, Irlenbusch e Sadrich (In Press) citam exemplos de trabalhos que destacam a sobreexploração de alguns recursos. Estas evidências têm-se tornado justificadas desde a descoberta de Gordon (1954 apud FISHER, IRLENBUSCH e SADRICH) que explica a tragédia dos comuns: quando o comportamento humano é levado à maximização de resultados individuais, e não pelo desejo de avanço social, é possível que todos, ao final, saiam perdendo.

$\mathrm{O}$ economista convencional entende que a escassez de um recurso pode ser medida através da trajetória do preço relativo deste recurso. Quanto maior for a escassez do recurso, maior será seu preço. Entretanto, existem diversas razões por traz da escassez que tornam este indicador, no mínimo, duvidoso (CONSTANZA e FOLKE, 1997 e GOUDER e KENNEDY, 1997, ambos apud BATABYAL, KAHN e O'NEIL, 2003). Hall e Hall (1984) enumeram seis destas razões: a) os preços observados são preços nacionais e, por isso, não refletem a oferta terrestre do recurso; b) os governos domésticos nacionais e internacionais distorcem os preços, através de políticas de incentivos ou de impostos; c) o mecanismo de mercado não é perfeito, de forma que o preço sofre influências do grau de monopólio; d) os recursos de propriedade comum ainda não foram completamente incorporados numa perspectiva de mercado, podendo-se encontrar diversos insumos desta natureza (água, por exemplo) que não

8 Utilizando-se do argumento da incerteza HowarTH (1996) demonstra a suficiência da condição de sustentabilidade forte no trato de questões de justiça intergeracional.

- "Degradação da camada de ozônio, aquecimento global, colapso da pesca, erosão dos solos e poluição do ar estão entre os sinais óbvios do desgaste ecológico" (BROWN et al, 2000 apud ANDRIANTIATSAHOLINIAINA, KOUIKOGLOW e PHILLIS, 2004), além de sinais de pobreza e doenças endêmicas. 
entram nos custos das firmas; e) a reciclagem desempenha um papel ainda mal definido na oferta total; f) existem diferentes tipos de escassez física.

Hall e Hall consideram que, de fato, todos estes pontos são importantes para enfraquecer o uso do preço como indicador. No entanto, alertam que é imprescindível que se reconheça, por exemplo, que se um governo impõe um imposto sobre a exploração de um recurso porque esta exploração está degradando a natureza, e afetando a qualidade do ambiente em desempenhar as suas duas outras funções, então este imposto faz parte do preço real do recurso e seu aumento reflete sim a escassez. Acima de tudo, eles reconhecem que o estoque de serviços ambientais limita o próprio fluxo de recursos naturais.

É para lidar com esta interdependência que se tem classificado os recursos por tipo de escassez fisica, destacando-se aqueles para os quais existe escassez ricardiana e aqueles para os quais existe a escassez malthusiana (SRIPADMINI, 2000). Malthus postulou um limite absoluto para os recursos, enquanto que Ricardo engendrou apenas uma qualidade decrescente aos recursos disponíveis. Estas postulações têm repercussões distintas sobre recursos renováveis e não-renováveis. ${ }^{10}$

Para recursos renováveis, a escassez malthusiana e a ricardiana estão baseadas no insumo terra; entretanto, o primeiro considerou o fato de ser este um insumo fixo e o segundo um insumo de qualidade continuamente decrescente. Sendo assim, tem-se, para Malthus, a lei dos retornos decrescentes e, para Ricardo, uma curva de custo marginal de longo prazo continuamente crescente.

Para os recursos não-renováveis, por sua vez, podem-se definir dois tipos de escassez para cada um desses economistas famosos (HALL e HALL, 1984). A escassez de estoque e a escassez de fluxo, de forma que se tem a escassez de estoque malthusiana e ricardiana (EEM e EER) e a escassez de fluxo malthusiana e ricardiana (EFM e EFR). Similarmente ao caso do recurso renovável, aqui a escassez malthusiana se aplica a recursos que apresentam uma qualidade uniforme, sem alteração; e a escassez ricardiana aos recursos com qualidade decrescente. ${ }^{11}$

\footnotetext{
${ }^{10}$ A aplicação dos conceitos de escassez malthusiana e ricardiana para recursos não-renováveis é uma extrapolaçāo conceitual de Hall e Hall (1984), já que Malthus e Ricardo referiram-se apenas ao fator terra.

"É da escassez Malthusiana, para o caso de recursos nāo-renováveis, que se originou o trabalho de Hotelling sobre escassez, hoje o mais citado na literatura (HALL e HALL, 1984).
} 
No caso de Malthus, pode-se considerar que a escassez de fluxo capta todos os elementos da escassez, dado que revela a existência ou não de reservas ainda não exploradas, já que a exploração, em Malthus, começa preferivelmente pela extensão. Os retornos decrescentes só começam a se revelar depois que todas as reservas entrarem em atividade. O preço para a escassez de fluxo malthusiana é igual ao custo médio acrescido valor presente do aumento no custo médio futuro e do custo de uso.

Para o caso de Ricardo, a situação é um pouco diferente, sendo a escassez de estoque a que revela todos os elementos da escassez. Em sendo um recurso de qualidade decrescente, novas reservas só passam a ser exploradas depois que as de melhor qualidades são exauridas ou estão muito perto da exaustão. O preço para a escassez de estoque ricardiana é igual ao custo médio mais o valor presente do aumento no custo médio futuro.

Mas estes preços sofrem as distorções observadas acima, de forma que se deve procurar por medidas mais precisas: o lado direito da equação. ${ }^{12}$ Ou seja, não se deve trabalhar com os preços, mas com os custos que lhes deveriam representar: custo médio, custo médio futuro, custo de uso, a depender da escassez referida.

A teoria econômica convencional da escassez conclui que $o$ resultado da indicação dos preços é uma inovação tecnológica que permite que o recurso que está se tornando relativamente mais caro possa ser poupado (BARNETT e MORSE, 1963 apud BATABYAL, KAHN e O'NEIL, 2003). A inovação tecnológica é então "induzida" pela dotação relativa, ou preços relativos, dos recursos. ${ }^{13}$

Um estudo feito por Managi et al. (2004) confirma a hipótese de que a evolução tecnológica tem de fato mitigado os efeitos da degradação ambiental. Mais do que isso, na verdade, os resultados do estudo permitem que eles concluam que a inovação tecnológica tem, na verdade, compensado a degradação nos anos mais recentes (entre 1995 e 2000). ${ }^{14}$

Esta inovação tecnológica é prevista atuar não somente sobre a substituição dos recursos, mas também sobre a diminuição da poluição, por exemplo. Nestes dois aspectos existe uma grande discus-

\footnotetext{
12 Além destes indicadores, existe uma forte vertente que caminha no sentido do desenvolvimento de indicadores físicos de escassez, ou sustentabilidade. Ver, por exemplo, GerbensLeenes at al (2003) e Bockstaller e Girardin (2003).

13 A solução do problema é encontrada endogenamente e é explicada pelo modelo de inovação induzida de Hayami e Ruttan para o caso da agricultura. Ver Sadoulet e de Janvry (1995).

${ }_{14}$ Eles estudam o período de 1995 a 2000
} 
são sobre que instrumentos de política utilizar para estimular, de forma mais eficiente, o avanço tecnológico. No que se refere à poluição, Parry (2003) endereça muito bem a questão, ele considera que, do ponto de vista da inovação tecnológica, é mais eficiente o controle da poluição do que estabelecer políticas de inovação tecnológica diretamente. Entre as políticas de controle da poluição, o autor defende as permissões de emissão negociáveis.

Mas o fato de os preços não darem o sinal mais apropriado da escassez dos recursos, como está sendo questionado neste momento, limita a ação da inovação tecnológica como parte resolvedora do problema. Sendo assim, os tomadores de decisão precisariam intervir impondo restrições reais no processo econômico e influenciando na pesquisa e inovação tecnológica. A questão estrutural aqui modifica o comportamento induzido do processo e é explicado pelo modelo de inovação induzida estrutural. ${ }^{15}$

A abordagem da escassez que apoiaria o argumento do tomador de decisão deveria ser fundamentada em uma análise que se preocupasse com a dependência fisica do ambiente como um todo, considerando sua multifuncionalidade. O resultado de uma análise deste tipo é que, em qualquer dado sistema, essas funções ambientais essenciais têm usos econômicos competitivos, o que torna os ativos ambientais relativamente escassos (BARBIER, 1989). Esse fato leva à necessidade de uma utilização irremediavelmente conscienciosa dos recursos, a qual esteja sempre buscando uma otimização do seu uso.

Então, a única forma de garantir a não extinção dos recursos ${ }^{16}$ é através de inovações tecnológicas que diminuam o uso dos recursos de uma forma geral, da energia, de geração de refugo, e que permitam melhorias técnicas no manejo ambiental (a exemplo de variedades de plantas melhoradas mais resistentes a secas e pragas). ${ }^{17}$ Estas inovações, segundo esta visão alternativa, ${ }^{18}$ só ocorrem com a decisão exógena de manter a base de recursos para as futuras gerações através de políticas macroeconômicas. ${ }^{19}$

Mas todas estas questões apresentadas até aqui versam sobre os recursos do ecossistema para os quais existe um mercado associado, muito embora muitas vezes estes sejam incompletos ou imperfeitos. Todavia, Batabyal, Kahn e O'neil (2003) chamam a atenção

\footnotetext{
is Para maiores detalhes ver Sadoulet e de Janvry (1995).

${ }^{16}$ O que é importante no caso da sustentabilidade forte.

${ }^{17}$ Se é que essas variedades não causam danos para a saúde humana.

${ }^{18}$ Barbier (1989) desenvolve o modelo completo.

19 Reconhecendo-se os já citados limites impostos pela fisica.
} 
para a necessidade de, também, monitorar a escassez do que ele chama de serviços do ecossistema, para os quais não existe um mercado associado e, portanto, não existe preço. Para estes, tem-se uma questão maior ainda.

Neste aspecto específico, a economia tem-se desenvolvido muito na atualidade, ${ }^{20}$ buscando dar valor a estes serviços. O desenvolvimento tem-se dado através do uso de técnicas de valoração ambiental, como a de valoração contingente, preços hedônicos ou custo de viagem. ${ }^{21}$ Todavia, como salientam Batabyal, Kahn e O'neil, a estimação deste valor, por si só, não significa a estimação de um indicador de escassez, a não ser que, como eles sugerem, se estabeleça uma associação entre a degradação e o bem-estar; fazendo-se assim, pode-se associar um valor de escassez com a degradação da qualidade ambiental. Aliás, nesse artigo, estes autores propóem uma estrutura teórica de controle estocástico para obter este valor numérico para o caso específico de um lago hipotético.

Nesta área de valoração de ativos ambientais degradados, sem preço de mercado, destacam-se ainda trabalhos que desenvolvem o que se chama de contabilidade verde. ${ }^{22}$ Cairns (2004), por exemplo, calcula o valor da degradação ambiental da atividade extrativa de uma mina de carvão. Carson at al. (2003) usam o método de valoração contingente para estimar as perdas com o vazamento de óleo provocado pela Exxon Vadley.

Dasgputa (2004) realiza um exercício de valoração das perdas na saúde que a comunidade pobre de Deli, na Índia, tem com a poluição da água, usando o método de valoração contingente. ${ }^{23} \mathrm{Os}$ resultados a que chega o autor podem ser comparados, segundo ele mesmo sugere, com os custos de se manter a água limpa, a partir do que se pode realizar uma análise de custo-benefício: é melhor realizar gastos com a compensação das doenças causadas ou realizar gastos com a limpeza das águas?

\footnotetext{
20 Ver o próprio Batabyal, Kahn e O'neil (2003) e Mota (1998).

${ }^{21}$ Isto para citar apenas os métodos de função demanda. Estas análises se utilizam, principalmente, de dados de seção cruzada; contudo, recentemente Swait at al. (2004) provaram que análises com séries temporais que levam em consideração a história dão resultados muito mais ricos.

2 A contabilidade verde contabiliza também a depreciação do capital natural.

${ }_{23}$ Quanto os indivíduos estavam dispostos a aceitar como compensação pela saúde danificada.
} 


\section{Considerações finais}

Este artigo buscou identificar os principais pontos que estão sendo discutidos dentro da disciplina de Economia dos Recursos Naturais, identificada como aquela que trata das questões relativas à função de ofertante de recursos que o meio ambiente desempenha. Entende-se, neste estudo, que as funções ambientais sejam completamente interdependentes, de forma que a Economia dos Recursos Naturais foi vista inserida através desta interdependência.

Entre os pontos de interesse na atualidade, tiveram destaque aqueles relevantes para garantir a equidade intergeracional; como o próprio conceito de sustentabilidade, a inovação tecnológica e os indicadores de escassez. Relativamente à eqüidade, relevou-se a sua diferença do conceito de eficiência, fato que tem provocado um grande desenvolvimento da teoria, adaptada até então para tratar apenas da eficiência. Apesar de já ser citada eventualmente anteriormente na teoria, a eqüidade foi um conceito quer surgiu com o desenvolvimento sustentável.

Tendo em vista a obtençāo da eqüidade, diferenciam-se os conceitos de sustentabilidade forte e sustentabilidade fraca, pelo fato de a primeira adotar uma visão mais conservadora com relação ao uso dos recursos naturais, ou seja, de sua total preservação para as gerações futuras. Todavia, identificou-se que, independentemente do conceito adotado, a mão do Estado deve ser forte no trato das questões ambientais, mesmo dentro dos limites da Economia dos Recursos Naturais.

A inovação tecnológica é identificada como uma arma de compensação da degradação de parte dos recursos ambientais. Instrumentos de estímulo a esta inovação foram discutidos, assim como a forma que a inovação deve tomar. Por fim, foram levantados aspectos dos indicadores de escassez, ou sustentabilidade dos recursos e serviços ambientais, relativizando-se a eficiência do indicador preço como um indicador de escassez. Neste contexto foram colocadas as diferenças entre escassez ricardiana e malthusiana.

Este trabalho se limitou a levantar as questões, sem entrar no mérito da maior parte delas, para o que fez uso de citações relativamente recentes, buscando apenas situar o interessado nas áreas de estudo que podem ser desenvolvidas. Foi limite também o campo da Economia dos Recursos Naturais, sem adentrar na área da Economia do Meio Ambiente ou da Economia Ecológica. 


\section{Referências bibliográficas}

ANDRIANTIATSAHOLINIAINA, L. C., KOUIKOGLOU, V. S. e PHILLIS, Y. A. 2004. Evaluating strategies for sustainable development: fuzzy logic reasoning and sensitivity analysis. Ecological Economics, 48, p. 149-172.

BARBIER, E. B. 1989. Economics, Natural-Resource Scarcity and Development: Conventional and Alternative Views. London: Earthscan.

BARNETT, H.J. e MORSE, C. 1963. Scarcity and Growth. Baltimore, MD: Johns Hopkins University Press.

BATABYAL, A A. KAHN, J. R. e O'NEILL, R. V. 2003. On the scarcity value of ecosystem services. Journal of Environmental Economics and Management, 46: p. 334-352.

BOCKSTALLER, C. e GIRARDIN, P. 2003. How to validate environmental indicators. Agricultural Systems, 76: p.639-653.

BROWN, L. R., FLAVIN, C. e FRENCH, H. 2000. State of the World 2000. New York: Norton.

BURTON, P. S. Intertemporal preferences and intergenerational equity considerations in optimal resource harvesting. Journal of Environmental Economics and Management: p.119-132, 2002.

CAIRNS, R. D. 2004. Green accounting for an externality, pollution at a mine. Environment and Resource Economics, 27, n. 4.

CARSON, R. T., MITCHELL, R. C., HANEMANN, M., KOPP, R. J., PRESSER, S. e RUUD, P. A. 2003. Contingent valuation and lost passive use: damages from Exxon Valdez oil spill. Environment and Resource Economics, 25, n. 3.

CONSTANZA, R., FOLKE, C. 1997. Valuing ecosystems services with efficiency, fairness, and sustainability as goals. In: Daily, G. C. Nature's Services. Washington DC: Island Press. DALY, H. E. 1995. On Wilfred Beckerrman's Critique of Sustainable Development. Environment Values. vol. 4: p. 49-55.

DALY, H. E. 1992. The economic growth debate: what some economists have learned but many have not. In: Markandya, A. e Richardson, J. (Eds.). Environmental Economics. London: Earthscan Publishers.

DASGPUTA, P. 2004. Valuing helth damages from water pollution in urban Delhi, India: a health production function approach. Environment and Development Economics, 9: p. 83-106.

FISCHER, M-E., IRLENBUSCH, B. e SADRICH, A. In Press. An intergenerational commom pool resource experiment. Journal of Environmental Economics and Management.

GERBENS-LEENES, P. W.; MOLL, H. C.; e UITERKAMP, S. 2003. Design and development of a measuring method for environmental sustainability in food production systems. Ecological Economics, 46: p. 231-248.

GOMES, G. M. 1995. Desenvolvimento sustentável no nordeste brasileiro: uma interpretação impopular. In: GOMES, G.M., SOUZA, H.R e MAGALHÃES, A.R. (orgs.) Desenvolvimento Sustentável no Nordeste. Brasilia: IPEA.

GOULDER, L. H. e KENNEDY, D. 1997. Valuing ecosystem services: philosophical bases and empirical methods. In: Daily, G. C. Nature's Services. Washington DC: Island Press. GORDON, H. S. 1954. The economic theory of a common property resource: the fishery: Journal of Political Economics, 62: p. 124-142.

GRUBB, M., KOCH, M., MUNSON, A., SULLIVAN, F. e THONSON, K.. 1993. The Earth Summit Agreements. London: Earthscan. 
HALL, D. C. E HALL, J. V. 1984. Conceitos e Medidas da Escassez de Recursos Naturais com um Sumário das Tendências Recentes. In: Journal of Environmental Economics and Management II, p. 363-379.

HOWARTH, R.B. e NORGAARD, R.B. 1994. Intergenerational Environmental Choices: A framework for analysis. Working Paper 94-6, Center for the Study of Global Transformations - UCSC

HOWARTH, R. B. 1996. Sustainability, Uncertainty, and Intergenerational Fairness. Forthcoming in FAUCHEUZ, S., VAN DER STRAATEN, J. e O'CONNOR, M. (eds.). Sustainable Development: Analysis and Public Policy. Dordrecht: Kluwer.

ICID. 1992. Sustainable Development: Concepts, Theories and Implications for Development Planning. Fortaleza: ICID.

KRYSIAK, F. C. e KRYSIAK, D. 2003. Production, consumption, and general equilibrium with physical constraints. Journal of Environmental Economics and Management, 46: p. 513-538

LÉLÉ, S. M. 1991. Sustainable Development: A critical review. World Development, vol. 19, n. 06: p. 607-621.

MANAGI, S., OPALUCH, J.J., JIN, D. e GRIGALUNAS, T. A. 2004. Tecnological change and depletion in offshore oil and gas. Journal of Environmental Economics and Management, 47: p. 388-409.

MARSDEN, T. 1994. Sustainable Rural Development: Toward a Research Agenda. Draft. MELO, A.S.S.A. 1999. A estimação de um índice de agricultura sustentável: uma aplicação para a área irrigada do Vale do Submédio São Francisco. Tese de Doutorado. Recife: PIMES/UFPE.

MOTA, R. S. 1998. Manual para Valoração Econômica de Recursos Ambientais. Brasília: Ministério do Meio Ambiente, dos Recursos Hídricos e da Amazônia Legal.

NAESS. A. 1990. Sustainable DEVELopment and DeEp ECology. IN: ENGEL, J. E ERGEL, J. (eDS). Ethics of Environment and Development. TUCSON: V. ARIZONA.

NALLE, D. J., MONTGOMERY, C. A., ARTHUR, J.L., POLASKY, S. e SCHUMAKER, N.H. In: Press. Modeling joint production of wildlife and timber. Journal of Environmental Economics and Management.

NOORGARD, R. B. 1990. Economic Indicators of Resource Scarcity: A Critical Essay. Journal of Environmental Economics and Management, vol. 19: p. 19-25.

PANDEY, R. e BHARDWAJ, G. 2004. Comparing the cost effectiveness of market-based policy instruments versus regulation: the case of imission trading in na integrated steel plant in India. Environment and Development Economics, 9: p. 107-122.

PARRY, I. W. H. 2003. On the implications of technological innovation for environmental policy. Environment and Development Economics, 8: p. 57-76.

PIERI, C., DUMANSKI, J., HAMBLIN, A e YOUNG, A. 1995. Land Quality Indicators, World Bank Discussion Papers, $n^{\circ}$ 315, The World Bank, Washington D.C..

PITTEL, K. 2002. Sustainability and Endogenous Growth. Edward Elgar Publishing.

RAMMEL, C. e VAN DEN BERGH, J. C. J. M. 2003. Evolutionary policies for sustainable development: adaptive flexibility and risk minimisin. Ecological Economics, 47: p. 121-133.

SADOULET, E. e DE JANVRY, A. 1995. Quantitative Development Policy Analysis. The John Hopkins University Press, Baltimore and London.

SHI, T. 2004. Ecological economics as a policy science: rhetoric or commitment towards an improved decision-making process on sustainability. Ecological Economics, 48: p.2336. 
SRIPADMINI, J. R. F. 2000. Natural Resource Scarcity (concepts and measurement). www.toenre.ac.in/seminar/s2rsp.htm, acessado em 9/05/05.

SWAIT, J., ADAMOWICZ, W. e VAN BUEREN, M. 2004. Choice and temporal welfare impacts: incorporating history into discrete choice models. Journal of Environmental Economics and Management, 47: p. 94-116.

SOUZA, N. J. (Coord). 1997. Introdução à Economia. São Paulo: Atlas.

STAATZ, J. M. E EICHER, C. K. Agricultural Development Ideas in Historical Perspective. In: EICHER, C.K. E J.M. STAATZ Agricultural Development in the Third World. The Johns Hopkins University Press, Baltimore e London, 1990.

TIETEMBERG, T. 1994. Environmental Economics and Policy. New York: Harper Collins College Publishers.

World Commission on Environment and Development. 1987. Our Common Future. New York: Oxford. 